\title{
Significance Aberrant Of CD133 Expression and Their Clinical Impact in Acute Lymphoblastic Leukemia among Sudanese Patients in Khartoum State
}

\author{
Ayman .E. Elnour Atia ${ }^{1}$, Tarig A. M. Hamid ${ }^{{ }^{2}}$, Mubarak Mustafa Elkarsany ${ }^{1}$, Samah Saif Eldin \\ M. Mohamed ${ }^{4}$, Nadia M. Madani ${ }^{1}$, Amro B. E. Bashir ${ }^{1}$ \\ ${ }^{1}$ Department of Hematology and Immunohematology, College of Medical Laboratory Science, Karary \\ University, Khartoum, Sudan \\ ${ }^{2}$ Department of Hematology and Immunohematology, Sharq El Nile College, Khartoum, Sudan \\ ${ }^{4}$ Department of Hematology and Immunohematology, Shaqra University, Riyadh, Saudi Arabia
}

*Corresponding author: Dr. Tarig A. M. Hamid; tarig24@hotmail.com

Received 09 August 2021;

Accepted 23 August 2021;

Published 01 September 2021

\begin{abstract}
Background: Acute lymphoblastic leukemia (ALL) is a malignant disease that arises from several genetic mutations in a single B- or Tlymphoid precursor, resulting in altered blast cell survival, proliferation, and maturation. Objectives: This current study was aimed to determine the significance of aberrant CD133 and 45 expressions in Sudanese Patients with Acute lymphoblastic Leukemia, and their clinical significance in Khartoum state. Materials and Methods: One hundred Sudanese participants were enrolled in this study as follows: 88 Patients diagnosed with ALL as a case and 12 healthy controls donors were randomly selected. 2.5-5 mL of EDTA blood/bone marrow for flowcytometry from each patient and controls subject. Results: Eighty-eight newly diagnosed Sudanese patients with acute lymphocytic leukemia (ALL) were involved in this study, their age average is (15.7) and their stander deviation (SD) is 17.4. The frequency of aberrant markers concerning control groups was significantly associated with all patients in CD 45 with a P value (0.0001), while there was no difference between cases and control in the frequency of CD 133 antigen, there was no significant difference between age groups in the expression of different aberrant antigens. The study was significantly more expressed CD133 in males than females. Also no significant difference in hematological parameters between patients with or without expression of Aberrant CD 45 and 133 markers, while we found significantly high TWBCs in T. cell leukemia. Conclusion: Aberrant myeloid antigens CD45 was significantly associated with childhood and adult ALL and may be considered as important prognostic factors, while CD 133 were not associated with ALL.
\end{abstract}

\section{Keywords: Acute lymphoblastic Leukemia, CD45, CD133, flow cytometer-Sudan}

\section{Introduction}

Acute lymphoblastic leukemia (ALL) is a malignant disease that arises from several genetic mutations in a single B or T-lymphoid precursor, resulting in altered blast cell survival, proliferation, and maturation. Eventually, this leads to the lethal accumulation of leukemic cells. The risk of developing ALL is more common in children younger than 5 years of age of 50 . Overall, about 4 out of every 10 cases of ALL are in adults ${ }^{[2]}$.

ALL is not common cancer, accounting for less than half of $1 \%$ of all cancers in the United States. The average person's lifetime risk of getting ALL is about 1 in 1000. The risk is slightly higher in males than in females and higher in whites than in African Americans ${ }^{[3]}$. Children may do better than adults because of differences like childhood and adult ALL, variances in treatment (children's bodies can often tolerate aggressive treatment better than adults), or some combination of these ${ }^{[4,5]}$.

The incidence of ALL is assessed at 1.6 per 100000 population, in 2016 alone, an estimated 6590 new cases were diagnosed, with over 1400 deaths due to ALL ${ }^{[6]}$. The incidence of ALL follows a bimodal distribution, with the first highest occurring in childhood and the second-highest occurring around the age of 50 [7]. While dose intensification strategies have led to significant progress in outcomes for pediatric patients, the prognosis for the 
elderly remains very poor. Even with a high rate of response to induction chemotherapy, only $30-40 \%$ of adult patients with ALL will achieve long-term remission ${ }^{[8]}$.

CD 45 Antigen also known as protein tyrosine phosphatase, receptor type C, also known as PTPRC is an enzyme in humans that is encoded by the PTPRC gene. PTPRC, which was originally called leukocyte common antigen (LCA) ${ }^{[9,10]}$. CD45 is a panleukocyte protein with tyrosine phosphatase activity involved in the regulation of signal transduction in hematopoiesis. CD45 does not colocalize with lipid rafts on murine and human nontransformed hematopoietic cells, but CD45 positioning within lipid rafts is modified during their oncogenic transformation to acute myeloid leukemia. CD45 colocalizes with lipid rafts on AML cells, which contributes to elevated GM-CSF signal intensity involved in the proliferation of leukemic cells ${ }^{[11]}$. Many alternatively spliced transcripts variants of this gene, which encode distinct isoforms, have been reported ${ }^{[9]}$. CD133 Antigen also known as prominin-1, is a glycoprotein in humans that is encoded by the PROM1 gene ${ }^{[9,10]}$. It is a member of pentaspan transmembrane glycoproteins, which specifically localize to cellular protrusions. While the precise function of CD133 remains unknown, it has been proposed that it acts as an organizer of cell membrane topology ${ }^{[12]}$. Today, CD133 is the most commonly used marker for the isolation of cancer stem cells (CSC). Its population derives from different tumors, mainly from various gliomas and carcinomas ${ }^{[13]}$.

\section{Materials and Methods}

The current study was a case-control study done in Khartoum State in the period from 2019 to 2021 . One hundred individuals were included in our study. Out of the One hundred, 88 patients were diagnosed with Acute lymphoblastic leukemia. Patients who had other forms of dysplastic disorders and tumors were excluded. 2.5$5 \mathrm{ml}$ of blood for flow cytometer was drawn in EDTA. The

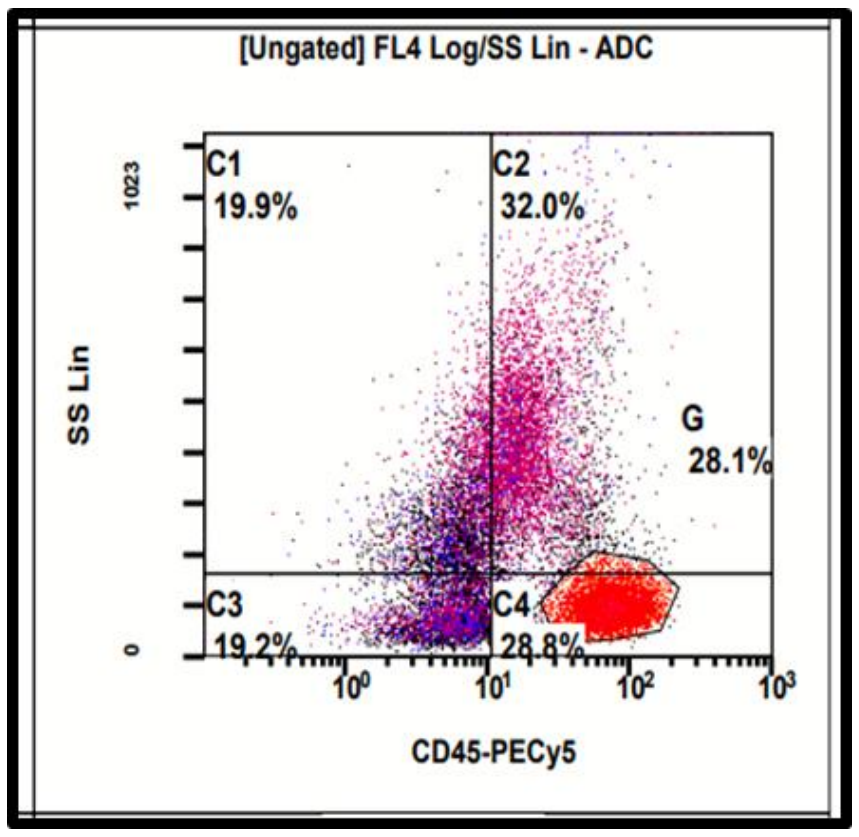

A: Control sample with negative result in CD 45

Eighty-eight participants were stratified by detection of aberrant CD markers (myeloid antigens), Frequency of each marker as illustrated in figure (2) which showed around $94.3 \%$ of the patients was CD45 positive and 30.7\% were positive for CD 133 . laboratory investigations included: A Complete blood count by using an automated hematology analyzer Sysmex kx21 and an Immunophenotyping by flow cytometry.

Immunophenotyping: All samples and healthy controls were analyzed using a flow cytometer (FC500 Beckman Coulter, Miami - USA), following the instructions of immune phenotyping kits offered by the IMMUNOSTEP Company.

Principle of flow cytometer: The underlying principle of flow cytometry is related to light scattering and fluorescence emission. Which occurs as light from the excitation source (commonly a laser beam) strikes the moving particles. The data obtained could give valuable information about biochemical, biophysical, and molecular aspects of particles. Light scattering is directly related to structural and morphological properties of the cell, while fluorescence emission derived from a fluorescence probe is proportional to the amount of fluorescent probe bound to the cell or cellular component.

Data were analyzed by using the Chi-square test and Fisher exact test. The numerical variables were presented in mean and stander deviation (SD). Two-sided P-values of $<0.05$ were considered significant. All the statistics were performed using the SPSS software version 20 and Graph Pad Prism software version 5.

\section{Results}

Eighty-eight ALL patients were involved in this study, the mean and SD age of patients were 15.7 and 17.4 years old, while the mean and SD of the control was 15.9 and 11.8.

The detection of aberrant $\mathrm{CD}$ markers was done by using flow cytometer techniques and the results were obtained in histograms representing the concentration of each aberrant antigens. Shown below is an example of a patient's results and control results summarized in figure (1)

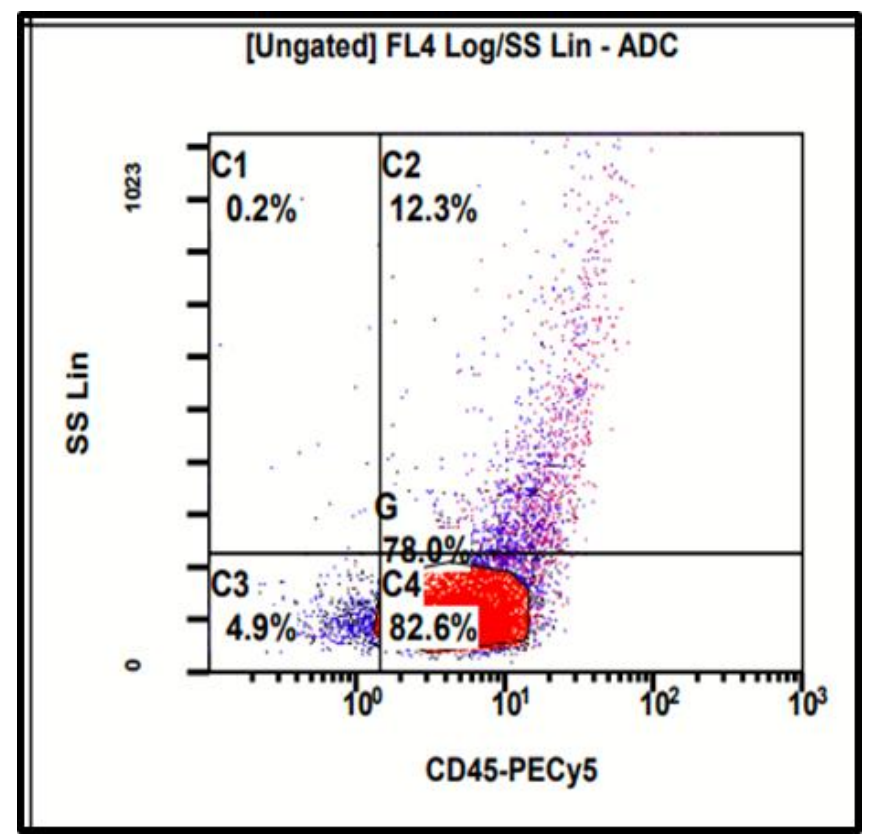

B: ALL sample with positive results for CD45

The distribution of the aberrant markers concerning control groups was significantly associated with All patients in CD 45 with a $P$ value (0.0001) (as shown in figure (3), while there was no difference between cases and control in the frequency of CD 133 antigen which statically not significant with $P$. value $(0.17)$. 


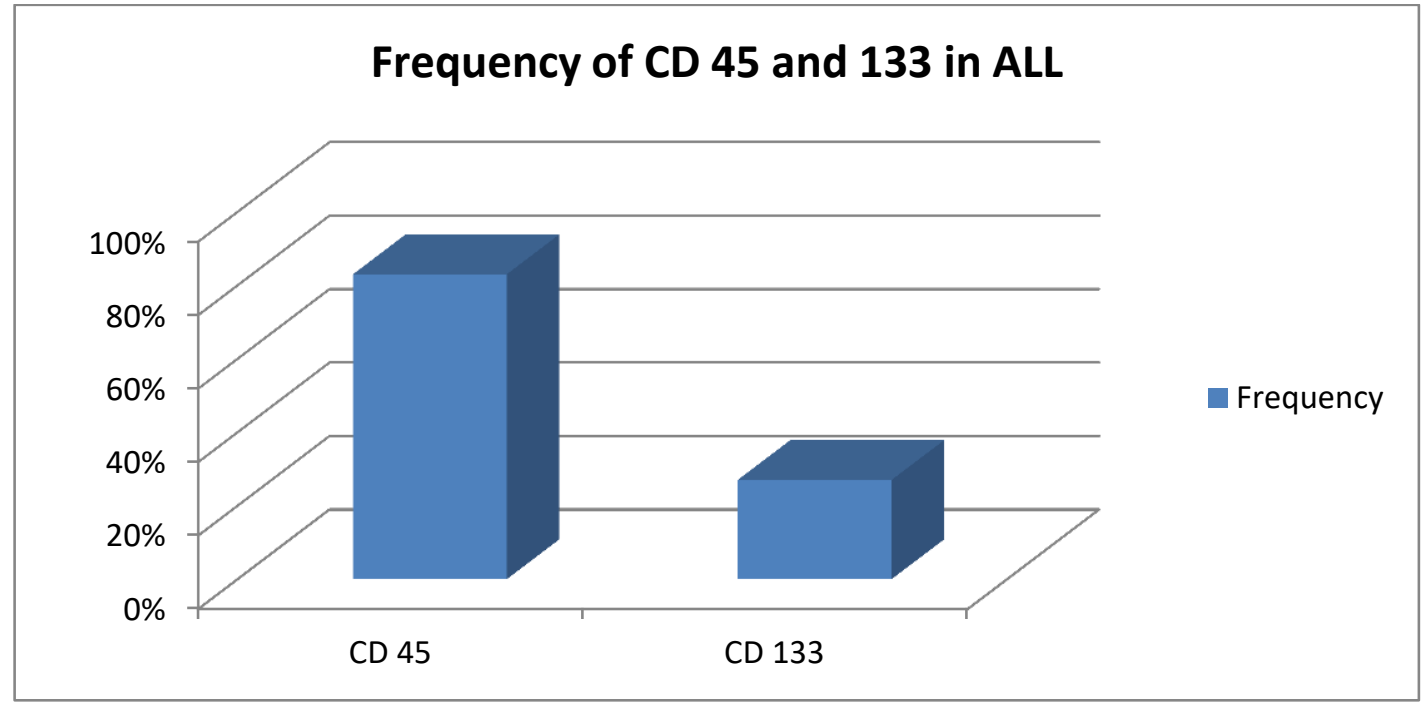

Figure 2: Frequency of CD45 and CD 133 makers in ALL patients

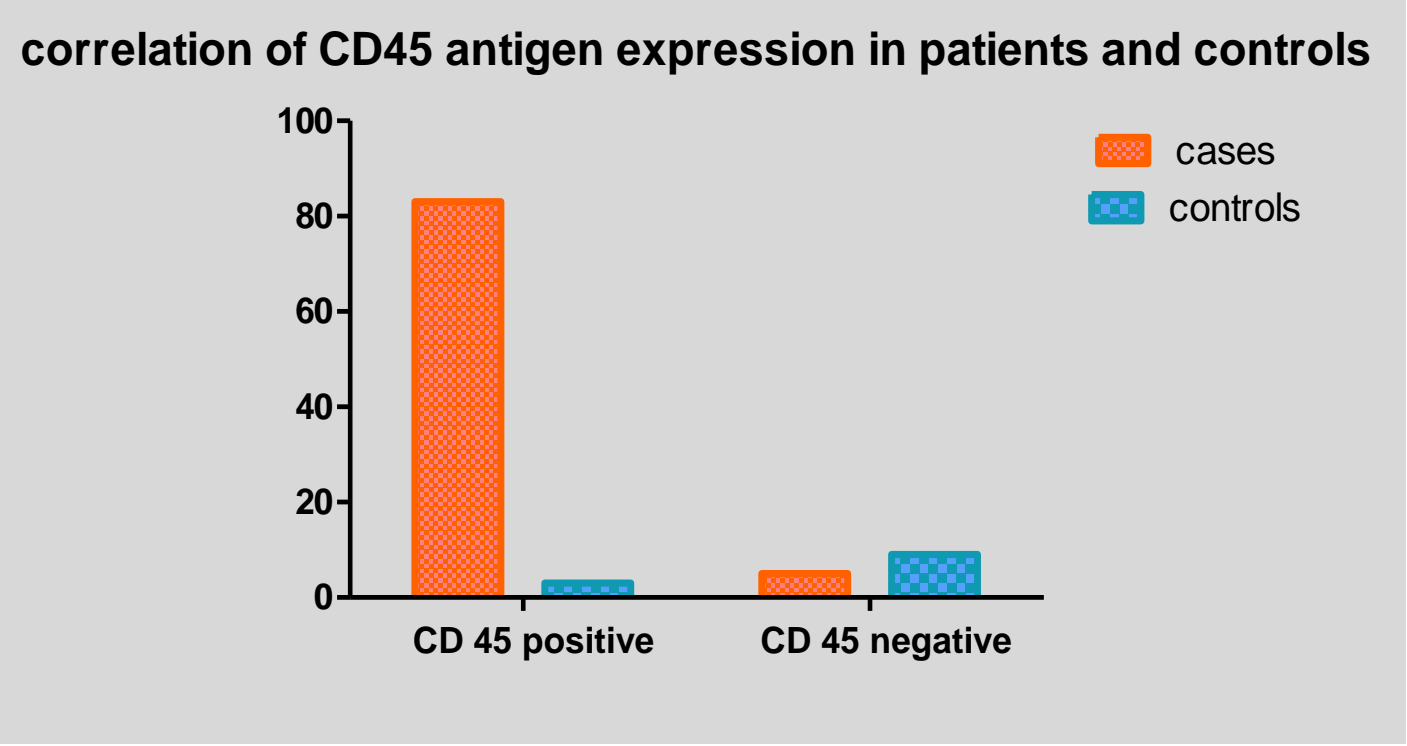

Figure 3: Correlation of CD 45 expression in case and control with a $P$ value $(0.0001)$

The general age distribution groups were shown in figure (4) were the most common group were between age $0-20$ that represent $79 \%$ of the whole study group, while the age distribution according to myeloid expression (CD45 and CD133) was shown in figure (5) with no significant between age groups in the expression of different aberrant antigens. The distribution of gender was more frequent male than female in cases and control group (figure 6)

\section{Age groups distribution among patients with ALL}

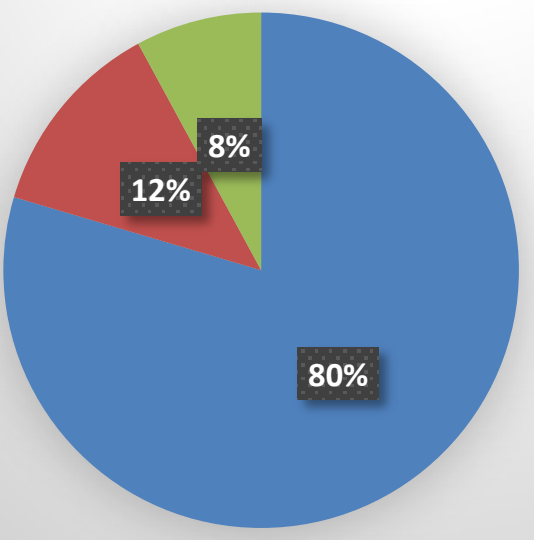

age 0 -20 years

- age 21-40 years

age $>40$ yesrs

Figure 4: Age groups distribution among patients with ALL 


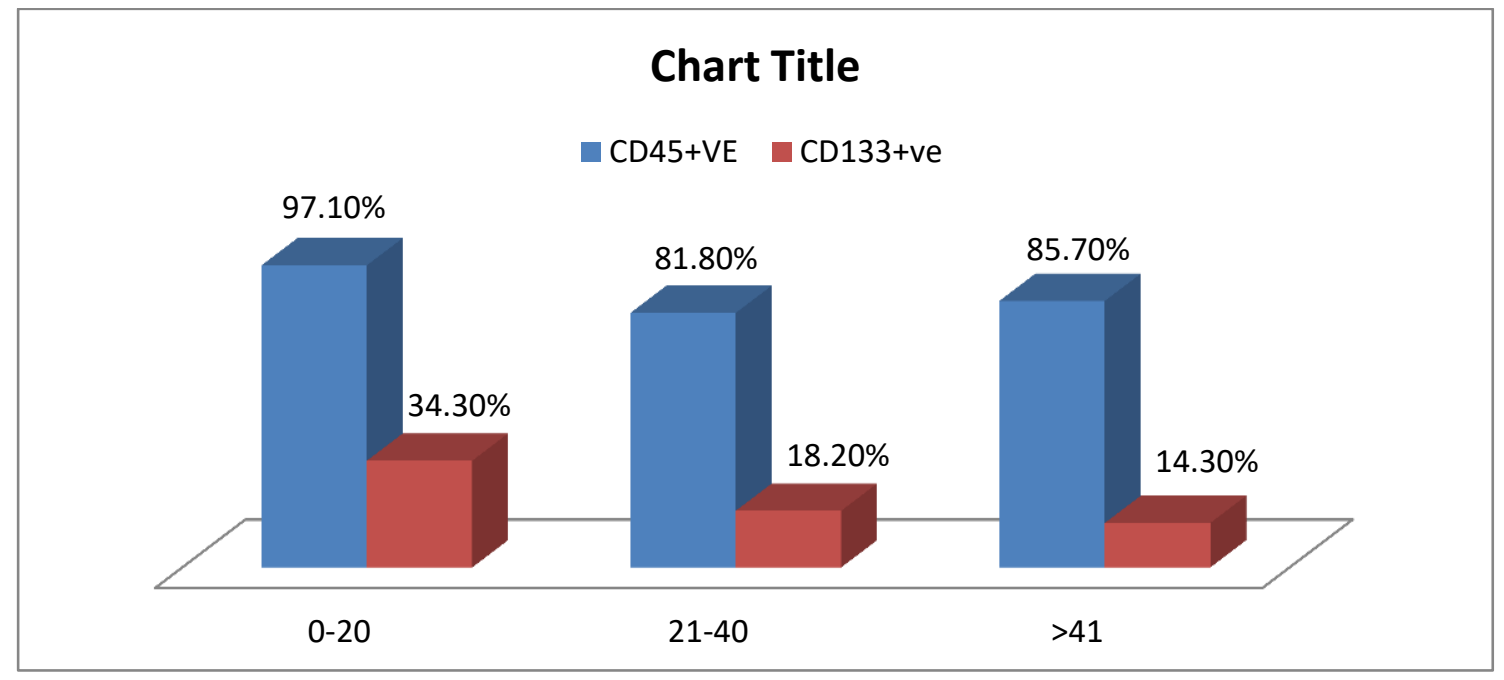

Figure 5: Frequency of aberrant myeloid expression in different Age

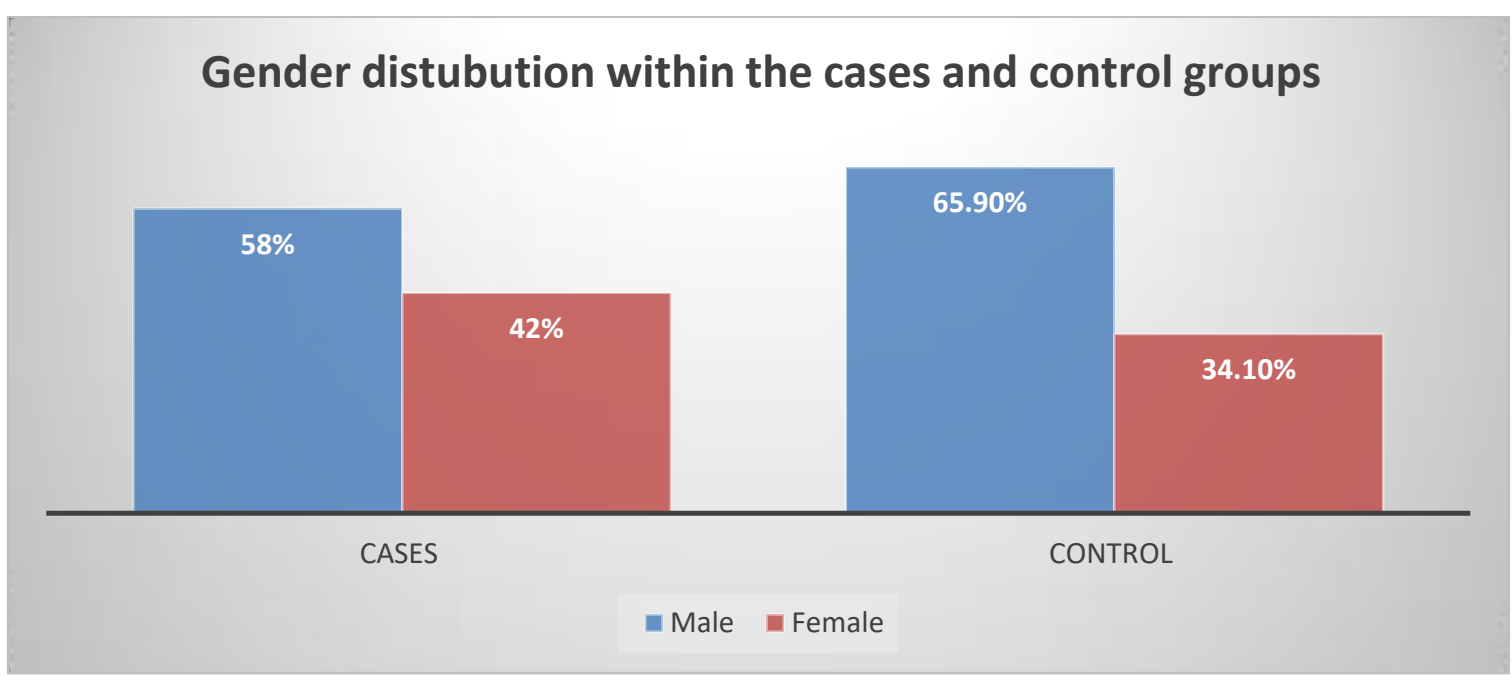

Figure 6: The gender distribution within case and control groups

The RBCs and platelets counts were insignificant decreased in most of $\mathrm{CD} 45+$ patients with p.value $(0.618$ and 1.000 , respectively) while the TWBCs was insignificant increased in most of CD 45+ patients with p.value (0.559). The RBCs and platelets counts were insignificant decreased in most of CD 133+ patients with p.value (and 1.000 and 0.128 , respectively) while the TWBCs was insignificant increased in most of CD 45+ patients with p.value (0.833).
The cases samples of the study group were also stratified for determined the immunologic classification into B or T-phenotypes. The panel of monoclonal antibodies that used was $\mathrm{CD} 2$, cytoplasmic (c) CD3, CD7 for precursor T-ALL, CD10, CD19, cCD79a for precursor B-ALL CD13, CD33, and cMPO, for myeloid lineage, while using HLA-DR and CD34 for non-lineage

On the other hand, the different profile was detected for childhood and adult B.cell and T.cell leukemia were summarized in figure (7 and 8).

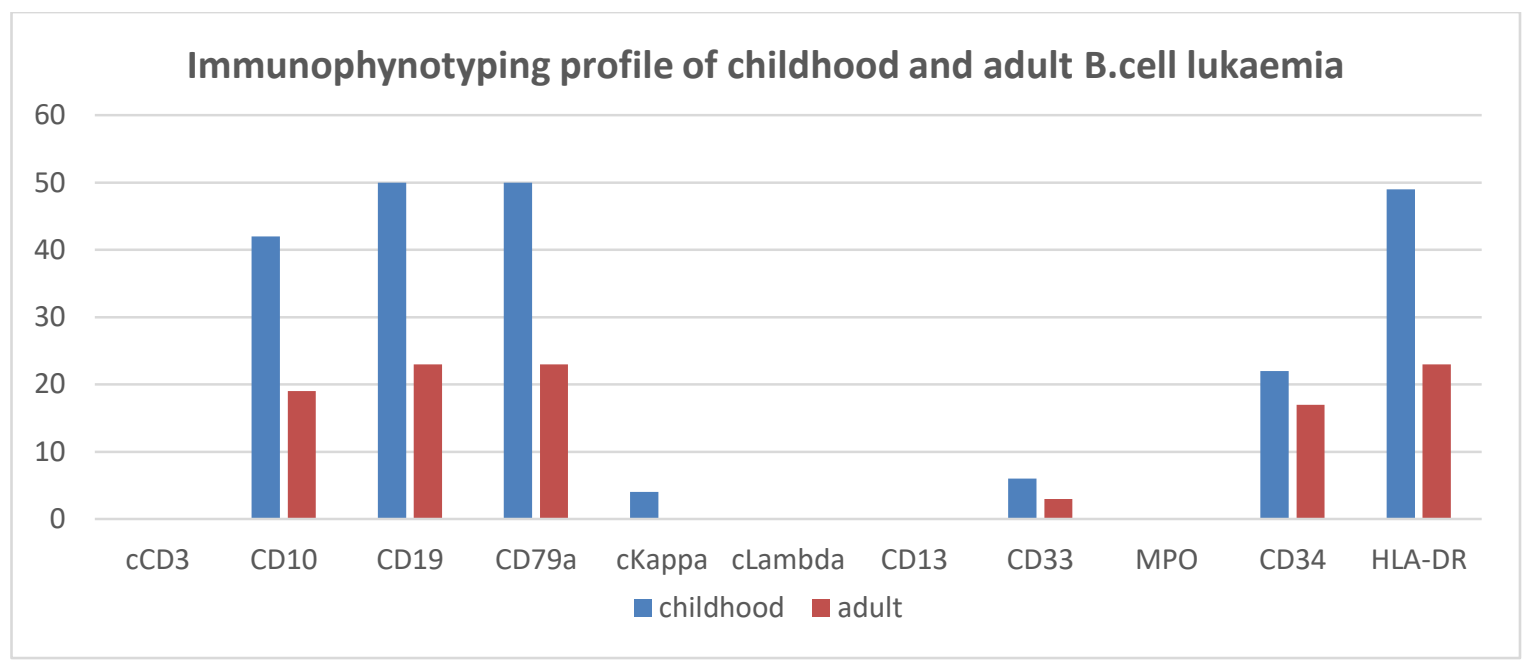

Figure 7: Frequency of aberrant antigens among different types of ALL 


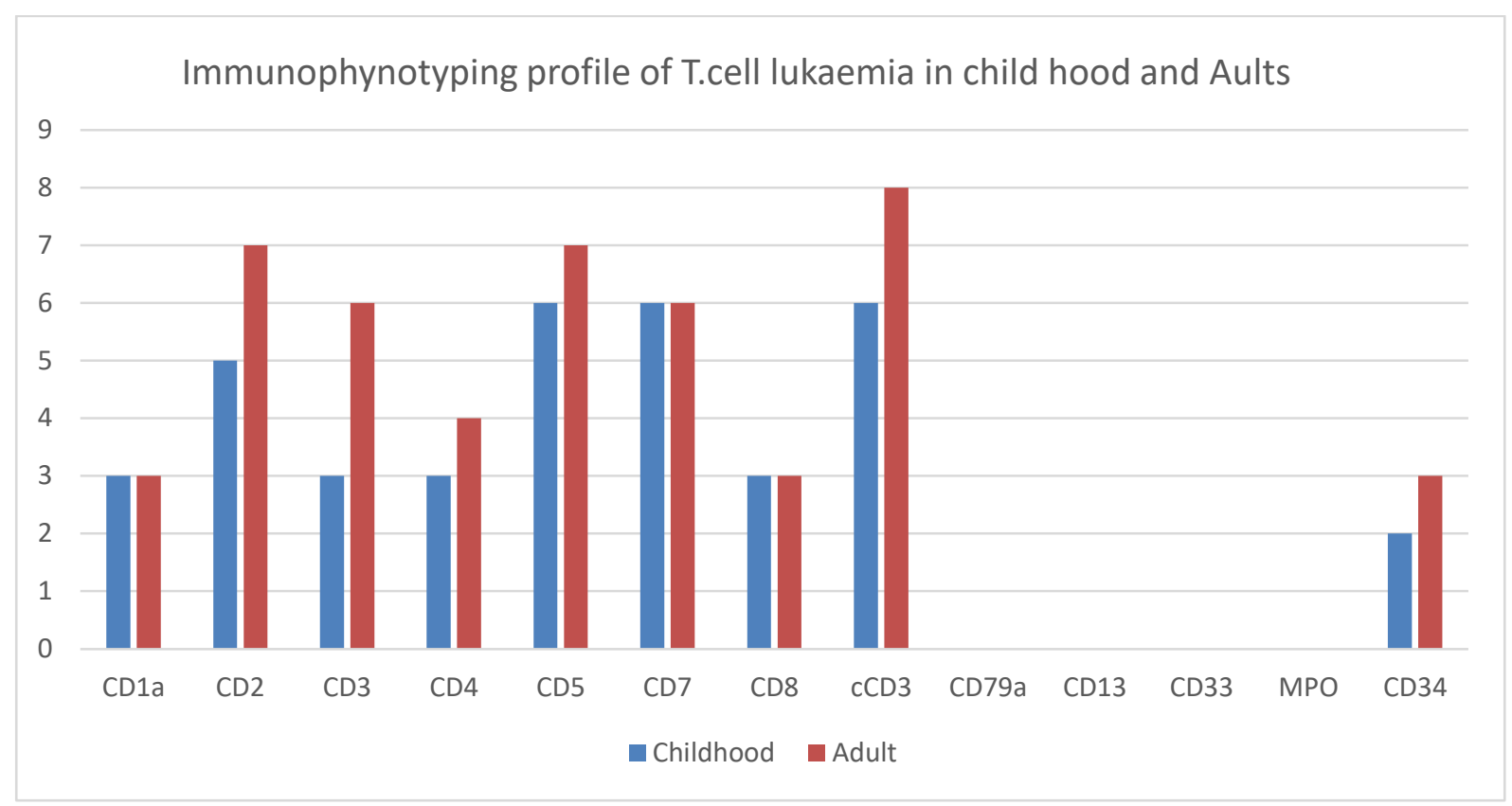

Figure 8: Frequency distribution of aberrant antigens among different types of T.ALL

The study group again were classified according to their origin into B. cell leukemia represents $84.1 \%$ (74 out of 88 ) while T. a cell represents $15.9 \%$ (14 out of 88 ) of the study group.

No significant difference in expression of all aberrant CD markers between B.cell leukemia and Tell leukemia groups where expressed in $94.6 \%$ and $92.8 \%$ respectively of each group and the frequency distribution of each maker in two types were illustrated in figure (9)

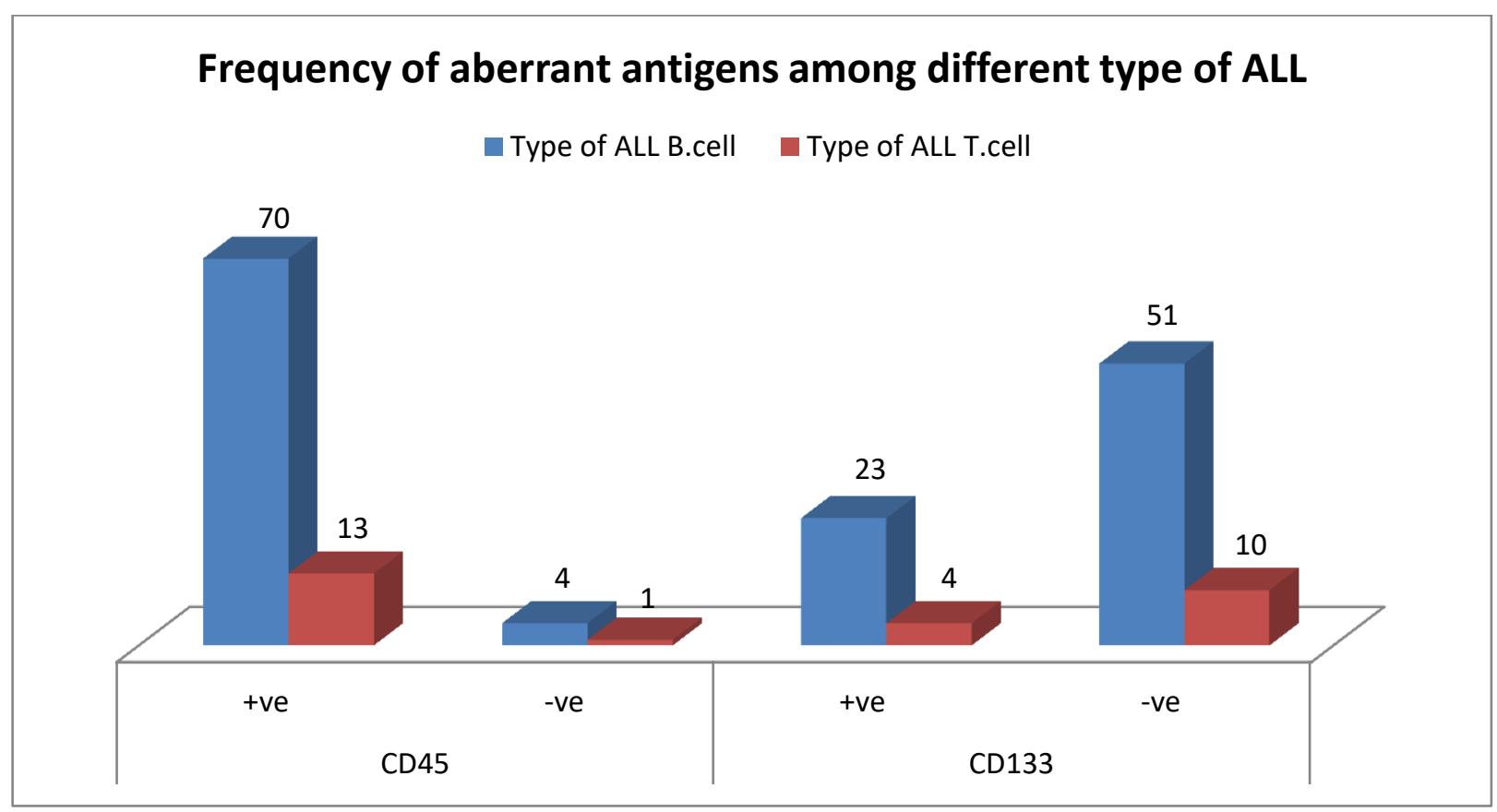

Figure 9: Frequency of aberrant antigens among different types of ALL

Further sub-classification of T.ALL was observed according to the result of flow cytometer and blood film in which they classified into (cortical and mature), where the B.ALL were classified into (early pre, pre, pro, and common), each sub-classification of B.cell and T.cell leukemia were compared with aberrant $\mathrm{CD}$ markers expression and summarized in tables ( 1 and 2 respectively).

Table 1: CD markers among different sub-classification of B.cell leukemia

\begin{tabular}{|l|l|l|l|l|}
\hline \multirow{2}{*}{ B. cell Stage } & CD45 count & CD133 count & -ve \\
\cline { 2 - 6 } & +ve & 1 & +ve & 31 \\
\hline EarlyPre B & 45 & 0 & 15 & 6 \\
\hline Pre B & 6 & 0 & 2 & 4 \\
\hline Pro & 6 & 3 & 4 & 12 \\
\hline Common & 13 & 4 & 21 & 53 \\
\hline Total & 70 & & \\
\hline
\end{tabular}


Table 2: CD markers among different sub-classification of T.cell leukemia

\begin{tabular}{|l|l|l|l|l|}
\hline \multirow{2}{*}{ T. cell Stage } & CD45 count & CD133 count \\
\cline { 2 - 5 } & +ve & -ve & +ve & -ve \\
\hline cortical & 6 & 0 & 3 & 3 \\
\hline Mature & 2 & 1 & 0 & 3 \\
\hline Total & 8 & 1 & 3 & 6 \\
\hline
\end{tabular}

There is no statistical significance of any clinical feature and aberrant CD 45 and CD 133 marker expression, the analysis results were shown in table (3)

Table 3: Association of common clinical feature and aberrant CD marker expression among the patient group:

\begin{tabular}{|c|c|c|c|c|c|c|c|}
\hline \multirow{2}{*}{\multicolumn{2}{|c|}{ Clinical features in ALL patients }} & \multicolumn{2}{|c|}{ CD45 } & \multirow[t]{2}{*}{ P.value } & \multicolumn{2}{|c|}{ CD133 } & \multirow[t]{2}{*}{ P. value } \\
\hline & & -ve & + ve & & -ve & + ve & \\
\hline \multirow[t]{2}{*}{ Fever } & yes & 3 & 70 & \multirow[t]{2}{*}{0.428} & 59 & 23 & \multirow[t]{2}{*}{0.196} \\
\hline & No & 2 & 13 & & 2 & 4 & \\
\hline \multirow[t]{2}{*}{ Lymphadenopathy } & yes & 1 & 48 & \multirow[t]{2}{*}{0.234} & 59 & 23 & \multirow[t]{2}{*}{0.828} \\
\hline & No & 4 & 35 & & 2 & 4 & \\
\hline \multirow[t]{2}{*}{ bleeding tendency } & yes & 0 & 12 & \multirow[t]{2}{*}{0.632} & 59 & 23 & \multirow[t]{2}{*}{0.710} \\
\hline & No & 5 & 70 & & 2 & 4 & \\
\hline \multirow[t]{2}{*}{ Hepatomegaly } & yes & 2 & 22 & \multirow[t]{2}{*}{0.888} & 59 & 23 & \multirow[t]{2}{*}{0.333} \\
\hline & No & 3 & 61 & & 2 & 4 & \\
\hline \multirow[t]{2}{*}{ Splenomegaly } & yes & 2 & 38 & \multirow[t]{2}{*}{0.801} & 59 & 23 & \multirow[t]{2}{*}{0.441} \\
\hline & No & 3 & 45 & & 2 & 4 & \\
\hline \multirow[t]{2}{*}{ Joint pain swelling } & yes & 1 & 28 & \multirow[t]{2}{*}{0.885} & 59 & 23 & \multirow[t]{2}{*}{0.238} \\
\hline & No & 4 & 55 & & 2 & 4 & \\
\hline \multirow[t]{2}{*}{ Cough } & yes & 0 & 7 & \multirow[t]{2}{*}{1.000} & 59 & 23 & \multirow[t]{2}{*}{0.248} \\
\hline & No & 5 & 76 & & 2 & 4 & \\
\hline
\end{tabular}

The present study showed the frequency of patients with positive with CD 45 and CD 133 together when correlated to gender were insignificant more frequent in males than a female with p.value (0.236) and no significant more frequent positive CD45 and CD133patients with B.cell ALL with p.value (0.689) as shown in table (5).

Table 5: Frequency of gender and type of ALL in CD45+ and CD133+

\begin{tabular}{|c|c|c|c|}
\hline & & CD45+ and CD133+ & P. value \\
\hline \multirow[t]{2}{*}{ Gender } & Male & 21 & \multirow[t]{2}{*}{0.236} \\
\hline & Female & 4 & \\
\hline \multirow[t]{2}{*}{ Type of ALL } & $\mathrm{T}$ cell & 4 & \multirow[t]{2}{*}{0.689} \\
\hline & B cell & 21 & \\
\hline
\end{tabular}

Also, these groups of co-expressed markers were compared with clinical feature and clinical feature severity and the result was illustrated in the table (6). The lymphadenopathy feature was also significantly related to co-expression with a $\mathrm{P}$ value (0.036), then again significant association between clinical feature and clinical feature severity with P.value (0.025) was detected.

Table 6: Frequency of clinical features and clinical feature severity

\begin{tabular}{|c|c|c|c|}
\hline \multicolumn{2}{|l|}{ Clinical features } & CD45+ and CD133+ & P.value \\
\hline \multirow[t]{2}{*}{ Fever } & No & 2 & \multirow[t]{2}{*}{0.337} \\
\hline & Yes & 8 & \\
\hline \multirow[t]{2}{*}{ Lymphadenopathy } & No & 1 & \multirow[t]{2}{*}{$0.036^{*}$} \\
\hline & Yes & 9 & \\
\hline \multirow[t]{2}{*}{ Bleeding tendency } & No & 10 & \multirow[t]{2}{*}{0.112} \\
\hline & Yes & 0 & \\
\hline \multirow[t]{2}{*}{ Hepatomegaly } & No & 7 & \multirow[t]{2}{*}{0.532} \\
\hline & Yes & 3 & \\
\hline \multirow[t]{2}{*}{ Splenomegaly } & No & 5 & \multirow[t]{2}{*}{0.491} \\
\hline & Yes & 5 & \\
\hline \multirow[t]{2}{*}{ Joint pain swelling } & No & 8 & \multirow[t]{2}{*}{0.594} \\
\hline & Yes & 2 & \\
\hline \multirow[t]{2}{*}{ Cough } & No & 8 & \multirow[t]{2}{*}{0.376} \\
\hline & Yes & 2 & \\
\hline \multirow[t]{2}{*}{ Clinical feature severity } & No & 1 & \multirow[t]{2}{*}{$\underline{0.025^{*}}$} \\
\hline & Medium & 9 & \\
\hline
\end{tabular}




\section{Discussion}

The frequency of aberrant myeloid antigen expression in acute lymphoid leukemia and their clinical significance is still not clear around the world. Currently, there are no published studies about the expression of these aberrant CD markers (CD45 and CD133) in acute lymphoblastic leukemia in Sudan. The current study found that the expression of aberrant markers CD45 was highly significant in cases of ALL. In comparison, the control group where CD45 was expressed in $94.3 \%$ of samples while only 30.7 $\%$ of samples expressed CD133. Earlier studies have also reported the frequency of different aberrant myeloid antigens in ALL in independent studies has varied from 10 to $100 \%{ }^{[14,15]}$ Similar to our results, the percentage of CD45 in the previous study was $78 \%$ in B.cell leukemia and $100 \%$ in T.cell leukemia ${ }^{[15]}$. While similarly agreeing with other previous studies, this indicates the important role of CD45 in ALL.

In contrast, our result of CD133 expression agrees with Fetnat M. Tolba and his colleague who reported no correlations were elicited between CD133 expression and ALL ${ }^{[16]}$, but disagreed with other studies that found strong expression of CD 133 in ALL [17]. These differences may be due to the detection of mRNA expression in their studies rather than flow cytometer.

Furthermore, the current study found the expression of CD 133 significantly expressed in males more than females, and no previous published study report that.

Additionally, the expression of this aberrant CD marker between childhood and adult (using $\leq 14$ as the cut-off for children) was done, and no significant difference between the expression of the three marker CD45 and CD133 in childhood and adult lymphoid leukemia was found. The expression agrees with a similar study that other aberrant markers in ALL have been reported an aberrant expression of CD133. The reported 39 and $23 \%$ cases were adults and children, respectively ${ }^{[14,18]}$.

Furthermore, a strong association of the presence of early prestage B.ALL leukemia in childhood rather than Adults. This association may indicate a good prognosis for patients which reported in other studies ${ }^{[19]}$

In this study, the classification of ALL into B-ALL and TALL also were compared with expression of aberrant myeloid antigen (CD45 and CD133), the frequency of these antigens was found (94.8\% and $31.2 \%$, respectively) of samples with B-ALL and (92.8\% and $28.5 \%$, respectively) in samples with T-ALL. Our results for $\mathrm{CD} 45$ were slightly higher than earlier reports where $83.2 \%$ samples with B-ALL and $28.3 \%$ samples with T-ALL were $\mathrm{CD} 45$ positive ${ }^{[20]}$ while disagreeing with other studies where report $100 \%$ samples with T-ALL and $28.3 \%$ samples with BALL are positive for aberrant markers ${ }^{[21]}$, this difference may be due to different study population and age factor since they carry their studies only on childhood patients.

Although there was no significant difference between the presence of B.ALL and T-ALL in their expression of these aberrant markers, with different clinical symptoms we found a strong association between expression of CD45 and lymphadenopathy in B-ALL, while in T-ALL, the cough was significantly associated with expression of CD133. These results disagree with the poor prognosis for CD 133 which was reported in a previous systemic review and meta-analysis by Wesley Messias Santos and et.al in Europe ${ }^{[22]}$. Other studies also report no clinical significance between CD133 and clinical feature presentation ${ }^{[16]}$.

Regarding the presentation of the clinical feature in our patients with ALL ${ }^{[23,24]}$, the most common clinical feature was fever $(82.9 \%)$ while the other feature represents $(55.7 \%, 45.5 \%$,
$33 \%, 27.3 \%, 13.6 \%$, and $7.9 \%$ ) for lymphadenopathy, splenomegaly, joint pain, hepatomegaly, bleeding tendency, and cough respectively. Similar findings were observed where Fever and hepatomegaly were the most common signs and symptoms in patients ${ }^{[25-27]}$. While in another study different results were reported hepatomegaly was the most common abnormality in almost $80 \%$, and $60 \%$ for both lymphadenopathy and splenomegaly ${ }^{[28]}$.

In agreement with a previous study from Oman ${ }^{[29]}$, the current study includes more males than females, this results in agreement with the facts previously mentioned in most papers ${ }^{[30-}$ ${ }^{32]}$.

Age destitution is an important role in the diagnosis and prognosis of acute lymphoid leukemia. In this study, we stratified the patients into four groups and the most common group between ages $0-20$ represent $79 \%$, this confirms the previous facts that Acute lymphoblastic leukemia is more common in children than adults ${ }^{[33,34]}$. Alternatively, no clinical significant correlation between age groups and expression of aberrant $\mathrm{CD}$ markers were found, which confirm the results from different earlier studies $[17,19,35]$.

B.ALL and T.ALL were detected in childhood and adult which occur in $68.9 \%$ and $31.1 \%$ of cases, while 42.8 . \% were B.ALL and $57.2 \%$ were T.ALL, respectively. Thus the B.ALL are more common in childhood than adults while the T.cell are more common in adults, an almost similar result was reported in an earlier study ${ }^{[36]}$. Alternatively, different immunophenotyping profiles were detected in adult B.ALL than in childhood profile. While in little similar profiles for both adult and childhood profiles in T.ALL were the same as what was reported in other studies $[14,18,36]$.

In the present study, patients with positive CD 45 and CD133 were compared according to the gender and type of ALL with no significant difference between male and female or between T.cell and B.cell leukemia in co-expression of aberrant CD markers were found. These results about co-expression disagreed with another study that reports co-expression of CD133 was associated with B.ALL ${ }^{[17]}$.

The co-expressed markers were compared with a clinical feature in addition to the significant association with lymphadenopathy as well as severe clinical remarks in B.ALL. Also, coughing was significantly associated with CD133 in T.ALL, this result confirms the hypothesis that co-expression of $\mathrm{CD}$ marker is associated with severe ALL that was reported in different studies ${ }^{[14,17]}$.

\section{Conclusions}

Ultimately, the present study concluded that there are no correlations between CD133 expression and ALL. Thus, we found the expression of CD 133 is significantly expressed in males more than females.

\section{Acknowledgements}

We gratefully acknowledge all clinicians participating in this study, we gratefully thank all staff of the flow cytometry center for their support. We would like to express our great thanks to DR; Osama Ali the general director of the flow cytometry center for their great effort.

\section{References}


[1] Pui, C.-H., Acute Lymphoblastic Leukemia, in Encyclopedia of Cancer, M. Schwab, Editor. 2011, Springer Berlin Heidelberg: Berlin, Heidelberg. p. 23-26.

[2] Atlanta, G., American Cancer Society. Cancer facts and figures 2013. American Cancer Society, 2013. 7(11).

[3] Pauly, M. and L.B. Silverman, Diagnosis and Treatment of Childhood Acute Lymphoblastic Leukemia, in Neoplastic Diseases of the Blood. 2018, Springer. p. 307335.

[4] Braithwaite, D., J. Demb, and L. Henderson, American Cancer Society: Cancer Facts and Figures 2016. Atlanta, GA: American Cancer Society, 2016.

[5] Street, W., Cancer Facts \& Figures 2019. American Cancer Society: Atlanta, GA, USA, 2019.

[6] Terwilliger, T. and M. Abdul-Hay, Acute lymphoblastic leukemia: a comprehensive review and 2017 update. Blood cancer journal, 2017. 7(6): p. e577-e577.

[7] Paul, S., H. Kantarjian, and E.J. Jabbour. Adult acute lymphoblastic leukemia. in Mayo Clinic Proceedings. 2016. Elsevier.

[8] Jabbour, E., et al., New insights into the pathophysiology and therapy of adult acute lymphoblastic leukemia. Cancer, 2015. 121(15): p. 2517-2528.

[9] Cortes, S.A., Automated analysis of high-throughput flow cytometry data from hematopoietic stem cell experiments. 2009, University of British Columbia.

[10] Kaplan, R., et al., Cloning of three human tyrosine phosphatases reveals a multigene family of receptorlinked protein-tyrosine-phosphatases expressed in the brain. Proceedings of the National Academy of Sciences, 1990. 87(18): p. 7000-7004.

[11] Saint-Paul, L., et al., CD45 phosphatase is crucial for human and murine acute myeloid leukemia maintenance through its localization in lipid rafts. Oncotarget, 2016. 7(40): p. 64785-64797.

[12] Irollo, E. and G. Pirozzi, CD133: to be or not to be, is this the real question? American journal of translational research, 2013. 5(6): p. 563-581.

[13] Kim, Y., et al., Cancer stem cell molecular markers verified in vivo. Biochemistry (Moscow), Supplement Series B: Biomedical Chemistry, 2017. 11(1): p. 43-54.

[14] Bhushan, B., et al., Aberrant phenotypes in childhood and adult acute leukemia and its association with adverse prognostic factors and clinical outcome. Clinical and experimental medicine, 2010. 10(1): p. 33-40.

[15] Sharma, R.K., et al., Aberrant myeloid antigen coexpression is correlated with high percentages of CD34positive cells among blasts of acute lymphoblastic leukemia patients: an Indian tertiary care center perspective. Blood research, 2014. 49(4): p. 241-245.

[16] Tolba, F.M., M.E. Foda, and D.A. Elshabrawy, Expression of CD133 in acute leukemia. Medical Oncology, 2013. 30(2): p. 1-8.

[17] Ji, H., et al., Aberrant expression of CD133 and CD82 in patients with pediatric acute lymphoblastic leukemia and the clinical significance. Oncology letters, 2017. 14(5): p. 5811-5818.

[18] Rezaei, M.S., et al., Characterization of immunophenotypic aberrancies in adult and childhood acute lymphoblastic leukemia: Lessons from Regional Variation. Iranian journal of pathology, 2020. 15(1): p. 1.

[19] Contreras Yametti, G.P., et al., Minimal Residual Disease in Acute Lymphoblastic Leukemia: Current
Practice and Future Directions. Cancers, 2021. 13(8): p. 1847.

[20] Ratei, R., et al., Immunophenotype and clinical characteristics of CD45-negative and CD45-positive childhood acute lymphoblastic leukemia. Annals of hematology, 1998. 77(3): p. 107-114.

[21] Nakamura, A., et al., Prognostic Impact of CD45 Antigen Expression in High-Risk, Childhood B-Cell Precursor Acute Lymphoblastic Leukemia: Children's Cancer and Leukemia Study Group (CCLSG). Leukemia \& lymphoma, 2001. 42(3): p. 393-398.

[22] Santos, W.M., et al., Role of New Immunophenotypic Markers on Prognostic and Overall Survival of Acute Lymphoblastic Leukaemia-A Systematic Review and Meta-analysis. European Oncology \& Haematology., 2019.

[23] Coustan-Smith, E., et al., Early T-cell precursor leukaemia: a subtype of very high-risk acute lymphoblastic leukaemia. The lancet oncology, 2009. 10(2): p. 147-156.

[24] Coustan-Smith, E., et al., Clinical importance of minimal residual disease in childhood acute lymphoblastic leukemia. Blood, 2000. 96(8): p. 2691-2696.

[25] Kohla, S.A., et al., Mixed Phenotype Acute Leukemia with Two Immunophenotypically Distinct B and T Blasts Populations, Double Ph (+) Chromosome and Complex Karyotype: Report of an Unusual Case. Clinical medicine insights. Blood disorders, 2015. 8: p. 25-31.

[26] Prajwal, M.S., K.K. Kundury, and M.J. Sujay, Assessing the awareness on occupational safety and health hazards among nursing staff of a teaching hospital. J Family Med Prim Care, 2020. 9(12): p. 5961-5970.

[27] Shahverdi, E., et al., Common presenting signs and symptoms in children with acute lymphoblastic leukemia. Basic and Clinical Cancer Research, 2020. 12(1): p. 26-33.

[28] Jaime-Pérez, J.C., et al., Revisiting the complete blood count and clinical findings at diagnosis of childhood acute lymphoblastic leukemia: 10-year experience at a single center. Hematology, transfusion and cell therapy, 2019. 41(1): p. 57-61.

[29] Al-Zaabi, M., et al., Impact of aberrant myeloid antigen expression on outcomes of patients with t-cell acute lymphoblastic leukemia. Oman medical journal, 2017. 32(3): p. 189.

[30] Mulder, R.L., et al., Health problems in survivors of childhood cancer: the need for international collaboration in long-term follow-up care. Future Oncology, 2013. 9(11): p. 1667-1670.

[31] Howlander, N., et al., SEER Cancer Statistics Review, 1975-2012, National Cancer Institute. Bethesda, MD, 2014.

[32] Singh, S.K., et al., A childhood acute lymphoblastic leukemia genome-wide association study identifies novel sex-specific risk variants. Medicine, 2016. 95(46).

[33] Foà, R., Acute lymphoblastic leukemia: age and biology. Pediatric reports, 2011. 3 Suppl 2(Suppl 2): p. e2-e2.

[34] Schwab, M., ALL, in Encyclopedia of Cancer, M. Schwab, Editor. 2011, Springer Berlin Heidelberg: Berlin, Heidelberg. p. 136-136.

[35] Dowd, A.A. and a.S. OM. Pattern and Age Distribution of Leukemia in Sudan-Retrospective Analysis. 2020. 
[36] Gupta, N., et al., Spectrum and immunophenotypic profile of acute leukemia: a tertiary center flow cytometry experience. Mediterranean journal of hematology and infectious diseases, 2019. 11(1). 